\title{
Kharash reaction on heterogenized metal complexes with bifunctional ligands
}

\author{
I. G. Tarkhanova ${ }^{1}$, A. I. Kokorin ${ }^{2}$, M. G. Gantman ${ }^{1, *}$, V. M. Zelikman ${ }^{1}$, D. S.Tsvetkov ${ }^{1}$ \\ ${ }^{1}$ M. V. Lomonosov, Moscow State University, Russia, Moscow \\ ${ }^{2}$ N. N. Semenov Institution of the Chemical Physics, RAS, Russia, Moscow
}

Email address:

Mikhail.gantman@gmail.com (M. G. Gantman)

\section{To cite this article:}

I. G. Tarkhanova, A. I. Kokorin, M. G. Gantman, V. M. Zelikman, D. S. Tsvetkov. Kharash Reaction on Heterogenized Metal Complexes with Bifunctional Ligands, American Journal of Physical Chemistry. Vol. 2, No. 2, 2013, pp. 24-32. doi: 10.11648/j.ajpc.20130202.11

\begin{abstract}
New catalytic systems for polyhalogenmethanes addition to olefins were obtained by immobilization of complexes of $\mathrm{Cu}, \mathrm{Co}, \mathrm{Mn}, \mathrm{V}$ (in the vanadyl form) and Fe with bifunctional ligands of aminoalcohols (monoethanolamine), substituted aminoalcohols (N, N-diethylaminoethanol), hydroxyaminoacids (serine, hydroxyproline) on the oxide supports. For $\mathrm{Cu}, \mathrm{Co}, \mathrm{Mn}, \mathrm{V}$ the extrem dependence of the catalyst activity on the surface concentration of metal was observed. For iron containing systems the mechanism of the process depends on the concentration of metal — at low concentration $(<0.4$ wt. \%) amine complex are active sites, at high concentration ( $>1.3$ wt. \%) — Lewis acid centres. By means of ESR the structure of active sites of the anchored complexes at different metal concentration was stated on the example of copper containing systems. Complexes isolated on the surface of mineral support at the expanse of strong covalent bonding of one of the lignd groups with surface hydroxyls were the most active. Such a structure provides the formation of the surface complexes of divalent copper with four nitrogen atoms in the coordination sphere.
\end{abstract}

Keywords: Transition Metal Complexes, Addition, Carbon Tetrachloride, Surface, Immobilization

\section{Introduction}

The addition of polyhalogenated methanes, $\mathrm{CCl}_{4}$ in particular, to olefins (Kharash reaction):

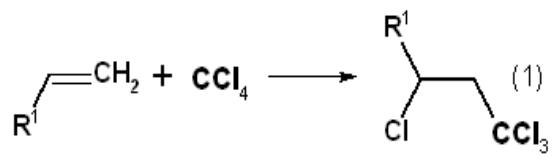

is one of the most effective ways of hydrocarbon modification. Although this reaction has been studied for many years [1-4], it is still of interest, as confirmed by recent publications [5-7]. The reaction (1) is usually performed in solutions in the presence of metal complex catalysts, initiators of various types. Copper [8], molybdenum [9], ruthenium [10], nickel [11] and iron [12] complexes can participate in (1) as the initiators. Another prospective use of reactions of polyhalogenmethanes with olefins, namely, the possibility to carry out so-called controlled polymerization, is of great interest nowadays. In this case, the process is held with an excess of alkene and aimed at obtaining polymers with high molecular mass and narrow molecular mass distribution. The distribution maximum depends on the reactant ratio. Diaminoaromatic nickel complexes and triphenylphosphine nickel or ruthenium complexes are used as catalysts of this reaction [13-17].

In most cases, the reactions between polyhalomethanes and olefins are performed in the presence of homogenous metal complex systems. Meanwhile, the advantages of using heterogenous catalysts are obvious, as this does not require catalyst separation, which is one the most laborous operations. Nevertheless, no effective and readily accessible hetergenous catalysts have been suggested for process (1) $[5,6,18]$. A few examples of catalysts of this type can be found among transition metal complexes grafted on silica surface with quaternary ammonium salts. These complexes can be obtained using organosilicon modifiers. However the difficulty of synthesis and high cost of the reagents make these catalysts not very promising for practical use.

This paper aims at working out cheap and useable effective and selective heterogenous catalysts of $\mathrm{CCl}_{4}$ addition to multiple bonds. It has been found that the catalysts can be easily obtained from bifunctional organic ligands containing simultaneously amino and hydroxyl 
groups, namely, hydroxyaminoacids and aminoalcohols. The condensation of the ligands with usual oxide supports gives rise to modified supports. These supports extract transition metal ions from solutions to form stable complexes able to catalyze the reaction of polyhalogenmethanes with olefins. Specifically, monoethanolamine (MEA, Ia $\mathrm{R}^{1}=\mathrm{H}, \quad \mathrm{R}^{2}=\mathrm{H}$ ), diethylaminoethanol (DEAE, Ib, $\mathrm{R}^{1}=\mathrm{C}_{2} \mathrm{H}_{5}, \quad \mathrm{R}^{2}=\mathrm{H}$ ), hydroxyproline ( $\mathrm{HPr}$ ) and serine (Ser Ic, $\left.\mathrm{R}^{1}=\mathrm{H}, \mathrm{R}^{2}=\mathrm{COOH}\right)$ can be used as the ligands. The reaction of $\mathrm{CCl}_{4}$ with oct-1-ene was studied as the model reaction for catalytic activity investigation.

To check the efficiency of the suggested technique, the catalysts of similar structure were obtained with the use of 3-aminopropyltriethoxysilane (APTES) as the organosilicon modifier [19]. A comparative analysis of two ways of the immobilization of complexes showed that the technique suggested in the previous paper yields more active catalysts.

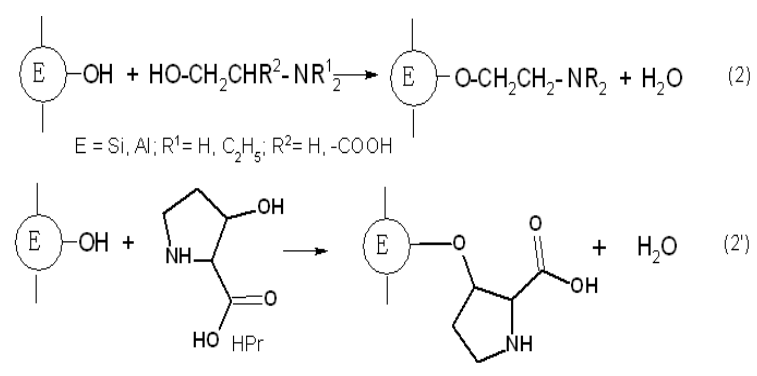

\section{Experimantal Part}

\subsection{Supports}

Silica gel KSK-2 had pores with specific volume $\mathrm{V}_{\mathrm{p}}=$ $1.0-1.25 \mathrm{~cm}^{3} / \mathrm{g}$; the specific surface area $\mathrm{S}=300 \mathrm{~m}^{2} / \mathrm{g}$; effective pore diameter $\mathrm{d}=12-15 \mathrm{~nm}$. Silica gel was washed with $0.1 \mathrm{M} \mathrm{HCl}$, dried and treated in a quartz ampoule under air flow at $450-500{ }^{\circ} \mathrm{C}$.

Silochrome $\mathrm{C}-120\left(\mathrm{~V}_{\mathrm{p}}=1.22-1.44 \mathrm{~cm}^{3} / \mathrm{g} ; \mathrm{S} \approx 120\right.$ $\mathrm{m}^{2} / \mathrm{g} ; \mathrm{d}=40-45 \mathrm{~nm}$ ) was washed with $0.1 \mathrm{HCl}$, dried and treated in a quartz ampoule under air flow at $200{ }^{\circ} \mathrm{C}$.

\subsection{Preparation of Catalysts}

Modification with MEA was performed from the gas phase by heating the silica gel in MEA vapor at $150-160^{\circ} \mathrm{C}$ for several hours followed by evacuation to a pressure of $\leq$ $10^{-2}$ Torr to remove the liberated water and excess alcohol [20]. DEAE was supported from toluene or xylene solutions by boiling for several hours with the continuous removal of water as an azeotropic mixture with the solvent. To support hydroxyproline and serine, the silica gel was impregnated with amino acid solutions in acetonitrile or tert-butanol; next, the solvent was removed in a vacuum, and the resulting samples were thermostatted at $150-180^{\circ} \mathrm{C}$ and then treated analogously to the samples modified with MEA [20].

To prepare immobilized complexes, we used metal acetonitrile solutions of chlorides $\left(\mathrm{CuCl}_{2}, \mathrm{MnCl}_{2}, \mathrm{CoCl}_{2}\right.$,
$\mathrm{FeCl}_{2}$, and $\mathrm{VOCl}_{2}$ ) [22-23]. In the synthesis of samples with various surface metal concentrations, modified silica gel was placed in acetonitrile solutions with different chloride concentrations and the resulting suspensions were magnetically stirred at $45-50{ }^{\circ} \mathrm{C}$ for $2 \mathrm{~h}$. Next, the silica gel was filtered off and washed with acetonitrile for the removal of an excess of metal salt. The concentration of organic matter in the resulting catalysts was determined by elemental analysis, and the concentrations of metals were determined by atomic absorption spectrometry.

\subsection{The Synthesis of Copper Complex with APTES}

The modification of KSK-2 with APTES was performed in the following way. A portion of KSK-2 was placed in a two-necked flask filled with toluene and equipped with a Dean-Stark trap, a reflux condenser, and a dropping funnel filled with the modifier. The reaction mixture was magnetically stirred and heated. The heating was finished when the volume of water condensed in the trap no longer changed. After that, APTES from the dropping funnel was added with heating and intense stirring. The reaction time was 6 hours. After that the modified support was washed three times with ethanol, filtered, and dried in air at room temperature. The immobilization of copper was performed as described earlier.

Individual metal complexes for comparison with heterogeneous catalysts were obtained in accordance with standard procedures [24-25]. MEA and Ser complexes were synthesized in ethanolic solutions, DEAE and $\mathrm{Hpr}$ complexes were obtained in acetonitrile solution.

\subsection{The Investigation of the Catalytic Complex Structure}

The compounds were studied by elemental analysis and IR spectroscopy. The samples for IR analysis were prepared in petrolatum. The spectra were recorded on an Infraflum FT-801 instrument. The Mössbauer spectra of ironcontaining systems were recorded at room temperature [23].

The evolution of copper and vanadyl ion containing catalysts was studied by EPR at $77 \mathrm{~K}$. The g-factors of complexes were determined by the measurement of hyperfine structure (HFS) components of $\mathrm{Mn}^{2+}$ ions in $\mathrm{MgO}$ simultaneously with the EPR spectra of copper ions. The number of paramagnetic centers in the test samples was determined by comparing the area under the absorption curve of a reference standard [20-22].

\subsection{The Catalytic Reactions}

The catalytic experiments were performed in sealed evacuated ampoules with intense stirring. The reaction mixture was separated and the products were analyzed by GLC-FID. The identification was based on the system of Kovats indices. The quantitative analysis was performed with the use of internal standard method. The activity of catalysts was determined from the initial rates of addition reactions in standard experiments: $0.2 \mathrm{~g}$ of a heterogeneous 
catalyst, $0.5 \mathrm{ml}$ of 1 -octene, and $4.5 \mathrm{ml}$ of $\mathrm{CCl}_{4}$ at $130^{\circ} \mathrm{C}$. The method of initial rates and the linearization of kinetic curves were used for calculating the kinetic parameters of the addition reaction. The reactant concentration was varied over the range of $\left[\mathrm{CCl}_{4}\right]$ from 0.4 to $1.25 \mathrm{~mol} / \mathrm{l}$; the catalyst weight was varied from 1.2 to $12 \mathrm{~g} / \mathrm{l}$. At low concentrations of $\mathrm{CCl}_{4}$, n-nonane was used as a solvent. To calculate the apparent activation energy, the reaction temperature was varied over the range $110-140^{\circ} \mathrm{C}$.

The activity of catalyst (A) was measured as the ration of initial rate to the mass of catalyst.

\section{Results and Discussion}

\subsection{Reaction Products}

The reaction of $\mathrm{CCl}_{4}$ with oct-1-ene in the presence of all studied catalysts at $100-130^{\circ} \mathrm{C}$ occurs selectively to form the addition product: 1, 1, 1, 3- tetrachlorononane. The product of Markovnikov addition, 1,1,1-trichloro- (2chloromethyl) octane, was not detected. The substitution products, chlorooctenes and 1,1,1-trichlorononane, were formed as minor products in amounts never exceeding $1.5-2 \%$ with respect to the yield of II. Other things being equal (reaction time, catalyst, and reactant amounts), the yield of II increased with increasing temperature. Traces of chloroform and hexachloroethane were formed. No telomerization products were found.

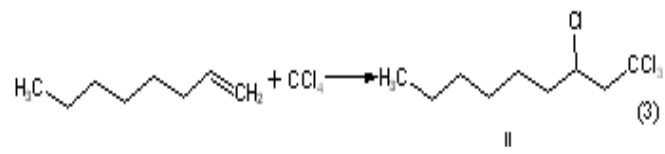

\subsection{The Influence of Heterogenization on the Catalytic Activity Of Metal Complexes}

The immobilization of catalysts on the surface of oxide support leads to a significant growth (up to an order of magnitude or more) of the catalytic activity with respect to individual complexes. This aspect of the process was studied in detail by the example of copper complexes. The activities of individual and heterogenized copper complexes at the same temperature and reactant concentrations are summarized in Table 1. The nature of the ligand influences slightly the activity of both homogenous and heterogenous catralysts. At the same time, heterogenization greatly increases the activity: TOF increases more than 10-fold. Indeed, the total amount of copper in the case of the heterogeneous catalyst was ten times lower than in the homogeneous catalyst, while the reaction yields, as follows from Table 1, was even higher under the commensurable conditions. Such a considerable growth of activity can be accounted for by a modification of complex structure upon immobilization. The modifications mentioned were studied by the example of amino alcohol copper complexes grafted on silica gel surface.

Table 1. The activity of attenuated and heterogenized copper complexes in the interaction between oct-1-ene and CCl $4 .\left(130^{\circ} \mathrm{C}\right.$, reaction time3 hours, $\left[\mathrm{CCl}_{4}\right]=9.1 \mathrm{M}$, [oct-1-ene $]=0.7 \mathrm{M}$ )

\begin{tabular}{|c|c|c|c|c|c|}
\hline \multirow{3}{*}{ Ligand } & \multicolumn{5}{|l|}{ Activity idices } \\
\hline & \multicolumn{3}{|c|}{ Attenuated complexes, $[\mathrm{Cu}]=2 \cdot 10^{-2} \mathrm{M}$} & \multicolumn{2}{|c|}{$\mathrm{SiO}_{2}-$ supported complexes $[\mathrm{Cu}]=2 \cdot 10^{-3} \mathrm{M}$} \\
\hline & $\begin{array}{l}\mathrm{L}: \mathrm{Cu} \text { ratio in the } \\
\text { complex }\end{array}$ & $\begin{array}{l}\text { Yield (II), \% of the } \\
\text { Theoretical amount }\end{array}$ & $\begin{array}{l}\text { Activity, TOF, } \\
\mathrm{mol} /(\mathrm{mol} \text { Cu hour) }\end{array}$ & $\begin{array}{l}\text { Yield (II), \% of the } \\
\text { theoretical amount }\end{array}$ & $\begin{array}{l}\text { Activity, TOF, } \\
\mathrm{mol} /(\mathrm{mol} \mathrm{Cu} \text { hour })\end{array}$ \\
\hline MEA & $2: 1$ & 56 & 6.5 & 85 & 100 \\
\hline DEAE & $1: 1$ & 48 & 5.6 & 64 & 75 \\
\hline $\mathrm{HPr}$ & $2: 1$ & 32 & 3.7 & 51 & 60 \\
\hline Ser & $2: 1$ & 39 & 4.5 & 75 & 88 \\
\hline
\end{tabular}

\subsection{The Structure of Heterogenized Copper Complexes}

According to quantitative analysis, surface modification by the above method allowed us to obtain silica gel containing 3-7 wt \% organic matter The processes of silica gel modification were studied in detail using silica gel samples with MEA as an example, we analyzed the surface concentration of free hydroxyl groups by IR spectroscopy (region of $3750 \mathrm{~cm}-1$ ) in the course of the catalyst synthesis.
The heat treated starting silica gel contained $3.6 \mathrm{mmol}$ of $\mathrm{OH}$ groups per gram, whereas after the reaction with MEA $(0.1 \mathrm{ml}$ per gram of $\mathrm{SiO} 2)$, it contained $0.23 \mathrm{mmol}$ of $\mathrm{OH}$ groups per gram. This indicates that most part of surface hydroxyls were etherified in accordance with (2). The chemisorbed amino alcohol molecules were not removed from the surface of silica gel after its treatment with a $\mathrm{CuCl} 2$ solution in acetonitrile: the concentration of hydroxyl groups in the prepared catalyst was $0.5-1 \mathrm{mmol}$ of $\mathrm{OH}$ groups per 
gram. Thus, the most part of $\mathrm{Si}-\mathrm{OCH} 2 \mathrm{CH} 2 \mathrm{NH} 2$ groups stayed invariable after the absorption of metal, and hydroxyl groups were not recovered for the most part.

The structure of the immobilized complex differs greatly from that observed in solution. The results of our EPR study illustrate well the structural difference between homogeneous and heterogenized copper complexes with DEAE. The $(\mathrm{CuCl} \cdot \mathrm{DEAE}) 2$ complex, either dissolved in $\mathrm{CCl} 4$ or isolated as a solid from solutions, does not have EPR signals, although divalent copper ions are present in this complex. It is most likely that the EPR-silent complex is binuclear with comparatively closely spaced copper ions; this is consistent with published data [26]. However, the complex prepared by the interaction of copper chloride with DEAE-modified silica gel exhibited an EPR spectrum characteristic of isolated $\mathrm{Cu} 2+$ ions (Fig. 1). The signal amplitude indicated that, at a copper concentration of $<0.1$ wt $\%$, at least $20-25 \%$ ions occurred as EPR-detectable mononuclear complexes.

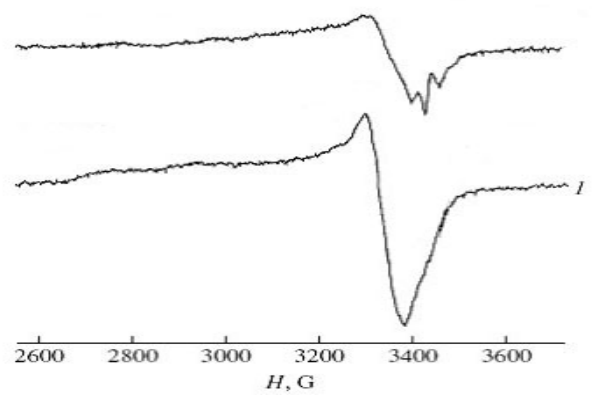

Figure 1. EPR spectra of copper(II) complexes with diethylaminoethanol supported on $\mathrm{SiO}_{2}$ at $[\mathrm{Cu}]_{0}=1.24$ (1) and 0.09(2) wt \%. T=77 K.

A similar situation is observed for MEA complexes. Figure 2 presents typical EPR spectra of $\mathrm{SiO}_{2}$-grafted copper complexes with MEA recorded at $77 \mathrm{~K}$. In this Figure, letters $\mathrm{A}, \mathrm{B}$ and $\mathrm{C}$ depict positions of EPR lines in the spectra of three different copper complexes correspondingly.

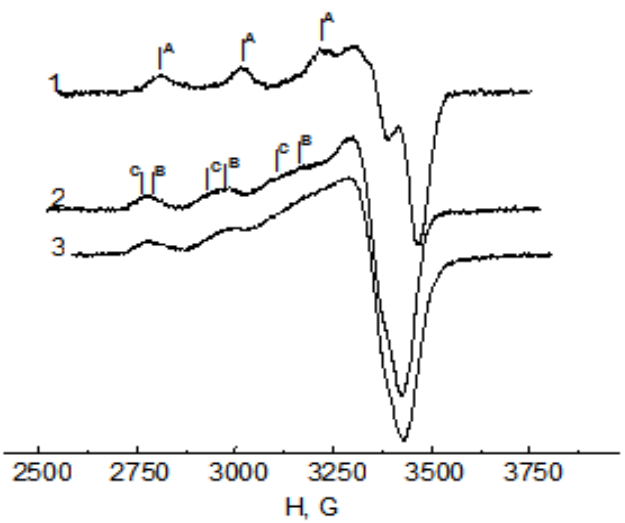

Figure 2a. EPR spectra $(T=77 \mathrm{~K})$ of copper(II) complexes with MEA supported on $\mathrm{SiO}_{2}$ at [Cu], wt \%: (1) 0.02, (3) 0.1. Spectrum 2 was obtained by subtracting spectrum 1 from spectrum 3.

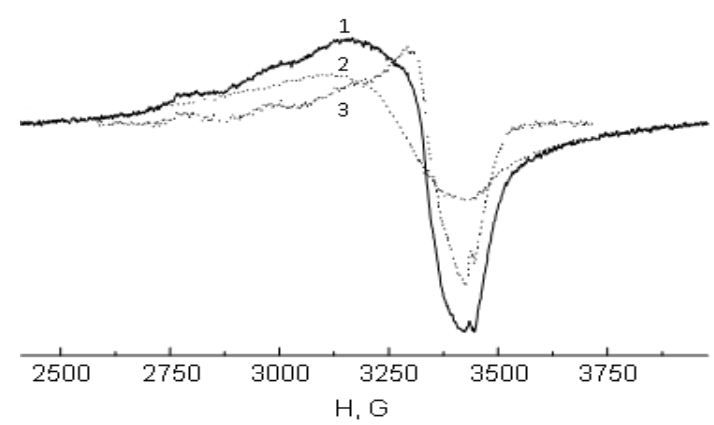

Figure $2 \boldsymbol{b}$. EPR spectra $(T=77 \mathrm{~K})$ of copper(II) complexes with MEA supported on $\mathrm{SiO}_{2}$ at [Cu], wt \%: 2.4. Spectrum (1) was represented as the superposition of singlet (2) and multiplet (3)

Figure 3 shows the dependence of the part of EPR-detectable copper ions $(\omega)$ on the copper content on the surface (wt. percent) for MEA and DEAE containing sy stems. It can be seen in Fig. 3 that at $[\mathrm{Cu}]_{0}<0.3 \mathrm{wt} \%$ for MEA complexes practically all copper ions in the sample were paramagnetic. As the total copper content increased $\left([\mathrm{Cu}]_{0}>0.3 \mathrm{wt} \%\right)$, the part of $\mathrm{Cu}(\mathrm{II})$ ions detected by EPR spectroscopy decreased, at that the amount of such ions remained approximately constant, whereas the amount of diamagnetic copper ions, which are EPR undetectable, increased. For DEAE we have slightly different situation: even at small concentrations the part of paramagnetic ions is about $20 \%$, upon growth of copper concentration the part of paramagnetic ions decreases.

At $[\mathrm{Cu}]_{0} \leq 0.3 \mathrm{wt} \%$, practically all copper complexes were detected by EPR spectroscopy, and the g-tensor parameters $\left(\mathrm{g}_{\|}>\mathrm{g}_{\perp}>2.000\right)$ determined from the spectra were characteristic of $\mathrm{Cu}$ (II) complexes with distorted octahedral structures (symmetry group $\mathrm{D}_{4 \mathrm{~h}}$ ) [27, 28]. At $[\mathrm{Cu}]_{0} \geq 0.3 \mathrm{wt} \%$, not only isolated copper(II) complexes but also associates with high local concentrations of $\mathrm{Cu}$ (II) ions occurred in the samples. Strong magnetic dipole-dipole and spin-exchange interactions between $\mathrm{Cu}$ (II) ions occurred in these associates and the fraction of associates increased with total copper concentration in the samples [29]. As it would be expected, the probability of formation of not only areas with high local concentrations but also $\mathrm{Cu}$ (II) complexes with structurally different ligand environments increased with the surface concentration of copper ions.

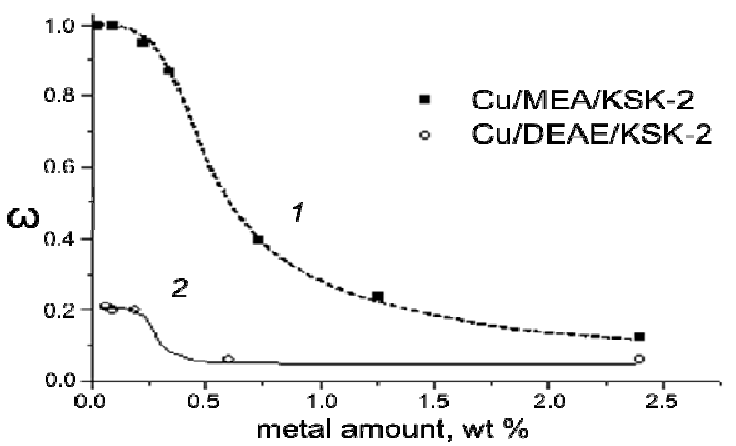

Figure 3. The dependence of the part of ESR detected copper complexes on the metal amount for $C u / M E A / K S K-2$ (1) and $C u / D E A E / K S K-2$ (2) 
On the increase in the copper surface concentration, the areas with high local metal concentration and copper (II) complexes with different ligand surrounding form more easily. This is manifested as broadening of hfs components of the EPR spectra and complication of the spectral pattern (Fig. 3). The spectrum in Fig. 2b is the superposition of the broad anisotropic singlet spectrum and the spectrum, whose form is similar to that of the spectrum 2 (Fig.2a), with consideration for the noise level of the experimental spectrum. It can also be seen in the Fig. 2a that the spectrum of a sample with 0.1 wt \% copper (spectrum 3) can be represented as the superposition of the spectrum of the sample with $0.02 \mathrm{wt} \%$ copper (spectrum 1) and spectrum 2 with an integrated intensity ratio between spectra 1 and 2 close to $1: 2.5$. In spectrum 2 , hfs components with a parallel orientation due to two types of complexes (B and C types) can be distinguished, which are different from an A-type complex in the magnetic-resonance parameters. Thus, even at a very low copper surface concentration on the support ( $\sim 0.1$ wt \%), different $\mathrm{Cu}(\mathrm{II})$ complexes with MEA occurred simultaneously in the samples.

The structural characterization of complexes A, B, and C was based on a comparison of the spin-Hamiltonian parameters found for these complexes with known published data. The parameters of complex $\mathrm{A}$ are close to the corresponding values of the $\left[\mathrm{Cu}\left(\mathrm{RNH}_{2}\right)_{4}\left(\mathrm{H}_{2} \mathrm{O}\right)_{2}\right]^{2+}$ tetraamine structures with nitrogen atoms in the equatorial plane of the polyhedron $\left(\mathrm{A}_{\|}=19.9 \pm 2.3 \mathrm{mT}, \mathrm{g}_{\|}=2.203 \pm 0.005\right.$, and $g_{\perp}=2.051 \pm 0.005$ ) [30]. It is likely that the high density of $\equiv \mathrm{SiOCH}_{2} \mathrm{CH}_{2} \mathrm{NH}_{2}$ groups grafted onto the surface of $\mathrm{SiO}_{2}$ is responsible for the occurrence of A-type structures, although their fraction is comparatively small.

The values of $\mathrm{g}_{\|}$and $\mathrm{A}_{\|}$for B-type complexes are close to those of $\left[\mathrm{Cu}\left(\mathrm{OCH}_{2} \mathrm{CH}_{2} \mathrm{NH}_{2}\right)_{2}\left(\mathrm{H}_{2} \mathrm{O}\right)_{2}\right]$ complexes, in which the $\mathrm{Cu}(\mathrm{II})$ ion is bound to the $\mathrm{N}$ atoms and - $\mathrm{O}-$ groups (deprotonated hydroxy groups) of two MEA molecules $\left(\mathrm{A}_{\|}=\right.$ $\left.18.8 \mathrm{mT} ; \mathrm{g}_{\|}=2.235\right)$ [31]. The difference in the values of $\mathrm{g}_{\|}$ is most likely due to the replacement of -O- groups or $\mathrm{H}_{2} \mathrm{O}$ in the coordination sphere of $\mathrm{Cu}$ (II) in the corresponding complexes in solution by the oxygen atoms of surface silanol groups. The values of $\mathrm{g}_{\|}$and $\mathrm{A}_{\|}$close to those of $\mathrm{B}$ complexes can also be observed in $\mathrm{Cu}$ (II) complexes with three amino group nitrogen atoms in the coordination sphere [28].

The parameters of a complex of the $\mathrm{C}$ type are very close to the values $A_{\|}=17.5 \mathrm{mT}$ and $\mathrm{g}_{\|}=2.269$, which were assigned to structures with two nitrogen atoms in the octahedral coordination environment of the copper ion [31]. Other sites in the coordination sphere may be occupied by the oxygen atoms of silanol groups and water adsorbed onto the surface of silicon dioxide. Probable structures of the mentioned complexes are presented in Fig. 4.
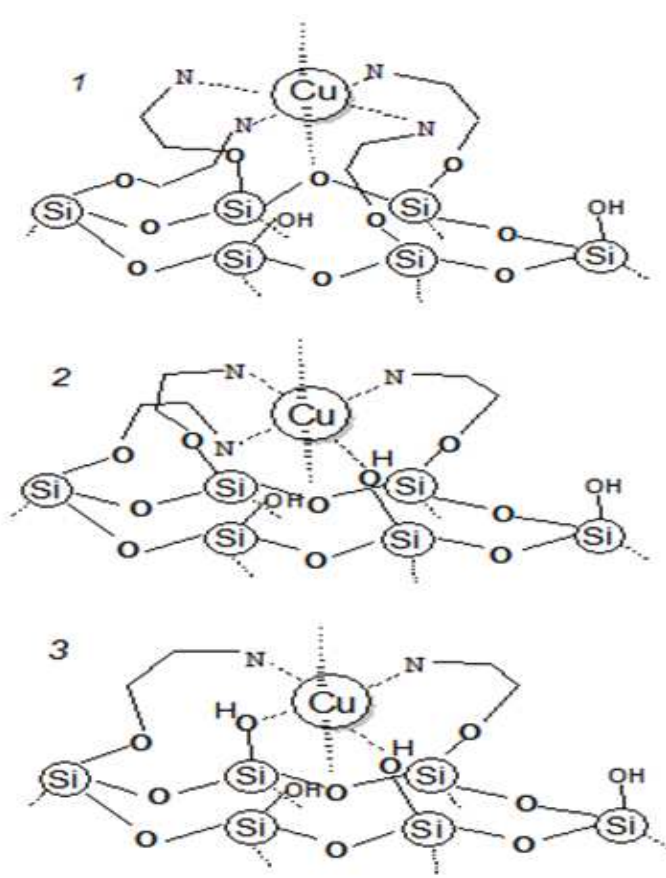

Figure 4. Probable structures of mononuclear complexes of types $A$ (1), $B$ (2) and C (3) on the silica gel surface.

\subsection{Kinetic Features of Ccl4 Addition to Oct-1-Ene in the Presence of Grafted Catalysts}

With an excess of $\mathrm{CCl}_{4}$, the reaction is described by a first-order rate equation. The determination of reaction order with respect to the catalyst and $\mathrm{CCl}_{4}$ by the method of initial rates allowed us to obtain the rate equation:

$$
-\mathrm{d}\left[\mathrm{CCl}_{4}\right] / \mathrm{dt}=\mathrm{k}_{\mathrm{obs}}\left[\mathrm{C}_{8} \mathrm{H}_{16}\right]\left[\mathrm{CCl}_{4}\right]
$$

where the obsereved rate constant $\mathrm{k}_{\mathrm{obs}}$ depends on the catalyst amount. Under the studied conditions, $\mathrm{k}_{\mathrm{obs}}$ is directly proportional to its mass.

In the studied temperature range $\left(110-140^{\circ} \mathrm{C}\right)$, the effective activation energy determined from the linearization of $\ln \left(\mathrm{w}_{0}\right)-1 / \mathrm{T}$ dependence was found to be $80 \pm 5 \mathrm{~kJ} / \mathrm{mol}$. A comparable value of the effective activation energy and analogous kinetic equation were obtained while studying $\mathrm{CCl}_{4}$ addition to styrene in the presence of catalysts based on ruthenium and molybdenum complexes. In such a systems so-called "coordination" mechanism takes place. [10]:

$$
\begin{gathered}
\mathrm{LCuCl}+\mathrm{L} \underset{\mathrm{K}_{1}}{\rightleftharpoons} \mathrm{L}_{2} \mathrm{CuCl} \\
\mathrm{L}_{2} \mathrm{CuCl}+\mathrm{CCl}_{4} \underset{\mathrm{K}_{2}}{\rightleftharpoons} \mathrm{L}_{2} \mathrm{CuCl} \cdot \mathrm{CCl}_{4} \\
\mathrm{~L}_{2} \mathrm{CuCl} \cdot \mathrm{CCl}_{4}+\mathrm{PhCH}=\mathrm{CH}_{2} \underset{\mathrm{k}_{1}}{\rightleftharpoons} \mathrm{L}_{2} \mathrm{CuCl}+ \\
\mathrm{PhCH}(\mathrm{Cl})-\mathrm{CH}_{2}-\mathrm{CCl}_{3} \\
\mathrm{~L}_{2} \mathrm{CuCl} \cdot \mathrm{CCl}+\mathrm{PhCH}=\mathrm{CH}_{2} \underset{\mathrm{K}_{3}}{\rightleftharpoons} \\
\mathrm{L}_{2} \mathrm{CuC} \cdot \mathrm{PhCH}(\mathrm{Cl})-\mathrm{CH}_{2}-\mathrm{CCl}_{3}
\end{gathered}
$$




\section{$\mathrm{L}_{2} \mathrm{CuCl} \cdot \mathrm{PhCH}(\mathrm{Cl})-\mathrm{CH}_{2}-\mathrm{CCl}_{3} \stackrel{\mathrm{k}_{2}}{\longrightarrow} \mathrm{L}_{2} \mathrm{CuCl}+$ $\mathrm{PhCH}(\mathrm{Cl})-\mathrm{CH}_{2}-\mathrm{CCl}_{3}$}

$\mathrm{L}$ is an organic ligand molecule

With the use of catalysts with different surface concentrations of immobilized copper complexes in the addition reaction, an extremal relationship between the activity and metal concentration was obtained (Fig. 5). The analogous regularities were observed for the catalysts, obtained with the use of APTES. As it can be seen from the figure at the equal surface concentration of copper the new catalysts, obtained using the procedure, suggested in the paper are more active than those obtaiend using APTES.

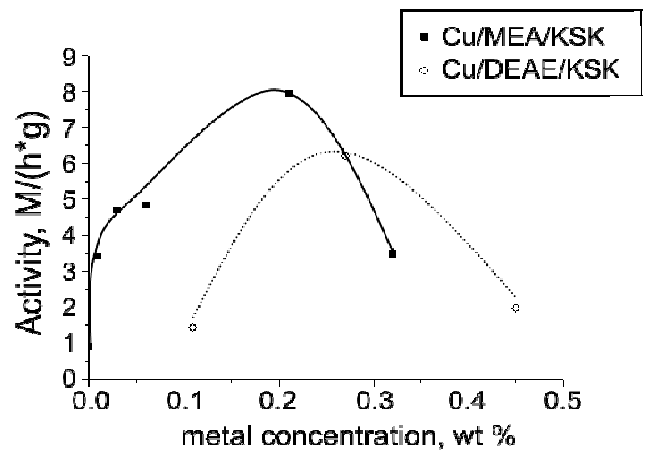

Figure 5. Dependence of the initial rates of $\mathrm{CCl}_{4}$ addition to 1-octene on the copper content of catalysts. $[\mathrm{Ol}]=0.6 \mathrm{~mol} / \mathrm{l} ;\left[\mathrm{CCl}_{4}\right]=9.3 \mathrm{~mol} / \mathrm{l} ; \mathrm{Vs}=0.5$ $\mathrm{ml}$; and $\mathrm{mCat}=0.02 \mathrm{~g}$.

Catalysts with low content of copper $(<0.3 \%)$ showed maximum activity. The increase in the catalytic activity together with a decrease in the metal content to $\sim 0.3 \mathrm{wt} \%$ can be accounted for by increase in the proportion of mononuclear complexes with higher nitrogen content in the coordination sphere.

During the reaction, the greater part of copper ions occur in the univalent state. As follows from Fig. 6, quite total $\mathrm{Cu}$ (II) reduction takes place in the very beginning of the reaction. After completion of the reaction and removal of the catalyst into air, the EPR spectrum appeared again.

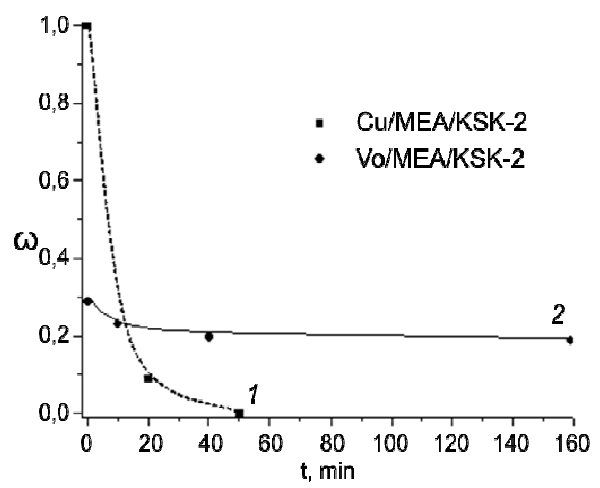

Figure 6. The change of the concentration of paramagnetic copper (1) and vanadium (2) complexes supported on $\mathrm{SiO}_{2}$ at $[\mathrm{Cu}]_{0}=0.3 \mathrm{wt} \%,[\mathrm{~V}]_{0}=0.17$ wt. $\%$ during the catalytic process at $130^{\circ} \mathrm{C} . \omega$ is the fraction of paramagnetic complexes with reference to the total metal content.

\subsection{The Influence of Metal Nature}

The data on the activity of catalysts based on $\mathrm{Cu}, \mathrm{Mn}, \mathrm{Co}$, $\mathrm{VO}$ are presented in Fig. 7.

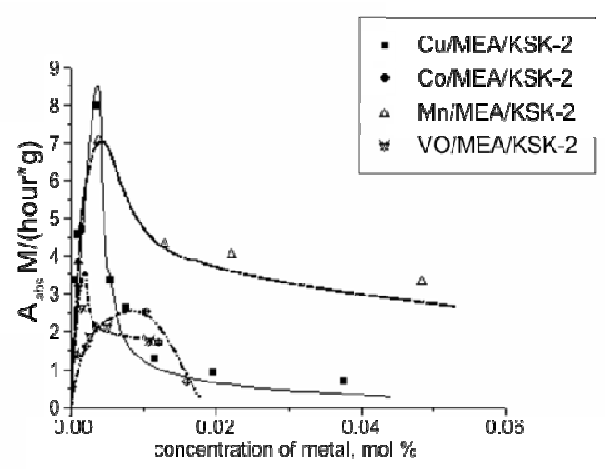

Figure 7. The dependence of the catalytic activity on the metal content in catalyst. 1- $C u / M E A / K S K-2,2-C o / M E A / K S K-2,3-M n / M E A / K S K-2$, 4$\mathrm{Ni} / \mathrm{MEA} / \mathrm{KSK}-2,5-\mathrm{VO} / \mathrm{MEA} / \mathrm{KSK}-2 ;\left[\mathrm{CCl}_{4}\right]_{0}=9.33 \mathrm{M},\left[\mathrm{C}_{8} \mathrm{H}_{16}\right]_{0}=0.64 \mathrm{M}$, $m($ cat $)=0.02 \mathrm{~g}, \mathrm{~T}=403 \mathrm{~K}$.

The extremal dependence of the activity on the metal content is observed for all metals except Fe. The activity maximum decreases in the following sequence: $\mathrm{Cu}>\mathrm{Mn}>$ Co $\sim$ VO. The regularities of the catalytic action of iron-containing systems differ dramatically from those for other metals and discussed in the next paragraph. All metals can be divided into two groups: highly-active ( $\mathrm{Mn}, \mathrm{Fe}, \mathrm{Cu}$ ) and low-active $(\mathrm{Co}, \mathrm{V})$. To study the reasons of such a different action of the metals the kinetics of transformations of vanadyle-containig systems in comparison with the copper-containing systems was studied.

On the fig. 8 ESR spectra of the VO/MEA/KSK-2 systems at various concentration of metal are given. All spectra are represented as a superposition of a multiplet, corresponding to isolated $\mathrm{VO}^{2+}$ ions, and of a broad singlet, typical for $\mathrm{V}^{4+}$ centres with high local concentration of metal (so-called associates), these ions are bonded with electron spin-exchange interaction. As the catalytic reaction of $\mathrm{CCl} 4$ with oct-1-ene proceeds the ESR spectrums slightly change, that can be accounted for by a slight decrease of total amount of paramagnetic vanadium complexes.

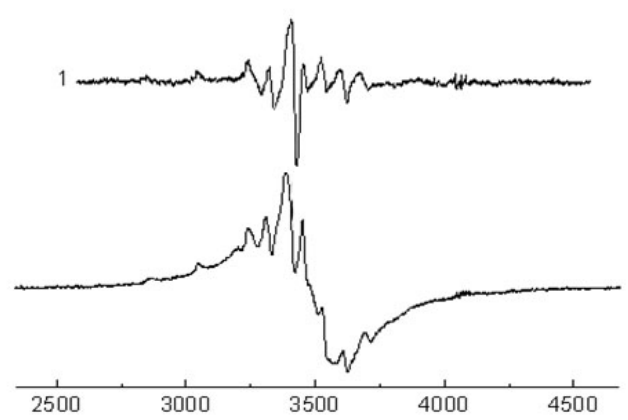

Figure 8. EPR spectra of paramagnetic complexes VO/MEA/KSK-2 at 0.07 (1) and 0.72 (2) wt. $\% T=77 \mathrm{~K}$ 
The fact that, the shape of the spectrum remains intact proves, that isolated complexes of vanadyl are very stable with respect to the components of reaction mixture, this causes low catalytic activity of these systems. We assume that the ability to change oxidation state easily and fast during the interaction with the componenets of the reaction mixture is a criterion of catalytic activity for all the metals.

\subsection{Regularities of Iron Catalyst Action}

The regularities of the catalytic action of iron differ dramatically from those mentioned above. The activity of the systems was found to be higher than that in systems with other metals. Its dependence on the metal content is shown in Fig. 7. The activity grows monotonically with increrasing amount of iron (Fig. 9).

In the region of low metal concentrations ( $\leq 0.3 \mathrm{wt} \%)$, the pattern of the curve is qualitatively identical to that in the presence of catalysts based on other metals. Differences manifest themselves in the region of higher $(>0.4 \mathrm{wt} \%)$ concentrations. At these metal concentrations, the activity of previously studied complexes decreased, whereas the growth of activity only somewhat slowed down in the case of iron; after reaching a critical metal concentration, the activity rapidly increased again. Under these conditions, as can be seen in Table 2, the composition of products changed: telomers and chlorooctene were formed along with the addition product.

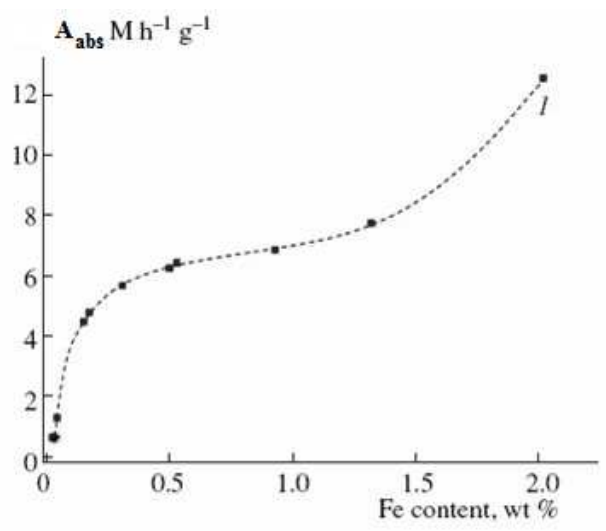

Figure 9. The dependence of the activity of $\mathrm{Fe} / \mathrm{MEA} / \mathrm{KSK}-2$ catalysts on the iron content.

Table 2. Comparison of the catalytic properties of Fe-containing systems

\begin{tabular}{lllll}
\hline Entry & Metal/ligand/support system & Metal content, wt \% & Total $\mathbf{C}_{\mathbf{8}} \mathbf{H}_{\mathbf{1 6}}$ conversion, \% & $\begin{array}{c}\text { Octene conversion } \\
\text { to telomers, } \%\end{array}$ \\
\hline 1 & & & & - \\
Yield of chlorooctene, \%
\end{tabular}

Note: ${ }^{a}$ The same sample after storage for a long time (see text).

Evidently, the observed differences are due to the presence of active sites other than those in the case of copper and other metals on the surface of Fe-containing catalysts. Based on published data, we can assume that iron chloride unbound to amino groups plays the role of these sites. We compared the activity and selectivity of action of the Fe/MEA/KSK-2 system and an analogous Fe/KSK-2 system (without MEA) at various metal concentrations. As can be seen in Fig. 8 and Table 2, only the samples containing MEA exhibited noticeable activity at low iron concentrations. As the iron content was increased, the activity of samples containing no MEA began to increase rapidly and became equal to the activity of the metal complex initiator starting with 1 wt $\%$.

The nature of active sites on the surface of the Fe/MEA/KSK-2 catalyst was studied by Mössbauer spectroscopy. The experimental spectrum is the superposition of signals from different compounds: two doublets and a singlet [23]. Components 1 and 2 (Table 3) correspond to absorption of $\mathrm{Fe}(\mathrm{III})$ in an octahedral environment. The isomer shifts and quadrupole splittings of these subspectra are close to the corresponding parameters of the spectrum of $\mathrm{Fe}(\mathrm{OH})_{3}$ [32]. The small deviation of the experimental parameters from the parameters given in the cited work is due to the fact that the symmetry of the environment of the iron ion is distorted by the partial replacement of $\mathrm{O}$ atoms by $\mathrm{Cl}$. A small isomer shift of the singlet line (component 3) allowed us to attribute this subspectrum in the Fe/MEA/KSK-2 system to the amine complexes of iron. 
Table 3. Isomer shifts ( $\delta$ ), qudrupole splittings ( $\Delta$ ), and line widths ( $\Gamma$ ) of the components of the Mössbauer spectrum of the Fe/MEA/KSK-2 catalyst (Fe content of $0.80 \mathrm{wt} \%$ )

\begin{tabular}{llllll}
\hline Subspectrum & $\boldsymbol{\delta}, \mathbf{~ m m} / \mathbf{s}$ & $\boldsymbol{\Delta}, \mathbf{m m} / \mathbf{s}$ & $\boldsymbol{\Gamma}, \mathbf{~ m m} / \mathbf{s}$ & Relative area, $\mathbf{~}$ & Reference [32] \\
\hline 1 (doublet) & $0.35 \pm 0.01$ & $0.60 \pm 0.01$ & $0.50 \pm 0.01$ & 45.26 & $\mathrm{Fe}(\mathrm{OH}) 3$ \\
2 (doublet) & $0.34 \pm 0.01$ & $1.00 \pm 0.00$ & $0.62 \pm 0.02$ & 43.23 & $\mathrm{Fe}(\mathrm{OH}) \mathrm{nCl} 3-\mathrm{n}$ \\
3 (singlet) & $-0.15 \pm 0.01$ & - & $0.27 \pm 0.01$ & 8.51 & $\mathrm{Fe} 3+-\mathrm{MEA}$ \\
\hline
\end{tabular}

Thus, iron completely occurs on the catalyst surface in a trivalent state. In this case, hydroxide or oxychloride species were predominant in samples with high metal concentrations. The oxidation of parent $\mathrm{Fe}(\mathrm{II})$ resulted from contact with atmospheric oxygen in the presence of (amine and acetonitrile) donors even in the course of catalyst preparation. At low iron concentration (0.35-0.40 wt. \%) mostly complexes of metal with grafted amino groups are present on the surface of the modified support.

The existence of amine complexes of iron is, by all appearances, the most important factor for the selective addition on Fe-containing catalysts. Note that the storage of the catalyst in air for several days changed its activity and selectivity (Table 2, entry 4). This was likely due to the gradual cleavage of hydrolytically unstable $\mathrm{Si}-\mathrm{O}-\mathrm{C}$ bonds on the surface of silica.

The behavior of catalysts prepared by supporting an aqueous solution of iron chloride onto unmodified silica was essentially different. A considerable portion of the metal passed into solution in the course of the reaction. The rapid increase in activity with increasing metal content (Table 2, entry 8-11) indicates that, in this case, unlike reactions with the participation of typical metal complex initiators, the formation of bridging and polymeric (in terms of the metal) structures not only does not interfere with the catalytic process but also, on the contrary, facilitates an increase in the activity.

It is likely that, at a high metal concentration, the reaction at Lewis acid sites was predominant; this reaction occurred by an ionic rather than radical mechanism. The processes of $\mathrm{CCl}_{4}$ in the presence of strong Lewis acids have long been known [33]. Based on published data we can propose the following scheme for the reaction catalyzed by iron(III) hydroxychloride, which is tentatively represented by the formula $\mathrm{Fe}(\mathrm{OH}) \mathrm{Cl}_{2}$ :

$$
\begin{aligned}
& 2 \mathrm{Fe}(\mathrm{OH}) \mathrm{Cl}_{2} \Leftrightarrow \mathrm{Fe}(\mathrm{OH}) \mathrm{Cl}^{+}+\mathrm{Fe}(\mathrm{OH}) \mathrm{Cl}_{3}^{-} \\
& \mathrm{Fe}(\mathrm{OH}) \mathrm{Cl}^{+}+\mathrm{CCl}_{4} \rightarrow \mathrm{Fe}(\mathrm{OH}) \mathrm{Cl}_{2}+\mathrm{CCl}_{3}^{+}
\end{aligned}
$$

Chain initiation (I) or

$$
\mathrm{Fe}(\mathrm{OH}) \mathrm{Cl}_{2}+\mathrm{CCl}_{4} \rightarrow \mathrm{Fe}(\mathrm{OH}) \mathrm{Cl}_{3}+\mathrm{CCl}_{3}^{+}
$$

Chain intiation (II)

$$
\mathrm{CCl}_{3}^{+}+\mathrm{Ol} \rightarrow \mathrm{R}_{1}^{+}
$$

Chain propagation
$\mathrm{R}_{1}^{+}+\mathrm{CCl}_{4} \rightarrow \mathrm{R}_{1} \mathrm{Cl}+\mathrm{CCl}_{3}^{+}$

Chain transfer, addition

$$
\mathrm{R}_{1}^{+}+\mathrm{Ol} \rightarrow \mathrm{R}_{2}^{+} \rightarrow \mathrm{R}_{\mathrm{n}}^{+}
$$

Chain propagation, telomerization

$$
\begin{gathered}
\mathrm{CCl}_{3}^{+}+\mathrm{Ol} \rightarrow \mathrm{R}_{1}^{\prime+}+\mathrm{CHCl}_{3} \\
\text { Chain propagation } \\
\mathrm{R}_{1}^{\prime++}+\mathrm{CCl}_{4} \rightarrow \mathrm{R}_{1}^{\prime \prime} \mathrm{Cl}+\mathrm{CCl}_{3}^{+}
\end{gathered}
$$

Chain transfer, substitution

Thus, the active sites of different nature are present in the catalysts prepared by the immobilization of iron chloride on the surface of silica modified with MEA. At a low metal content (to $0.4 \mathrm{wt} \%$ ), the reaction occurred with high selectivity on amine complexes by a coordination mechanism. In samples with high metal concentrations, iron hydroxychlorides, which form Lewis acid sites, were responsible for catalysis. Under these conditions, the rate of reaction was high; however, telomers and chlorooctene were formed along with the addition product. Thus, varying the metal concentration in the catalyst can be considered a convenient and effective means for regulating the direction and rate of reactions of polychloroalkanes with unsaturated compounds.

\section{Acknowledgments}

This work was supported by Russian Foundation for the Basic Research and Darville Enterprises Limited

\section{References}

[1] M.S. Kharash, E.V. Jensen, W.H. Urry (1947) J. of Am. Chem. Soc., 69: 1100

[2] Mayo F.R. (1948) J. Am. Chem. Soc., 70: 3689

[3] Freidlina R.Kh., Chukovskaya E.C. (1974) Synthesis, 10: 447

[4] Assher M., Vofsi D. (1963) J. Chem. Soc., 1887

[5] T.Sasaki, M.Tada, C.Zhong (2008) J. Mol. Cat. A, 279: 200-209

[6] Yohey Oe, Yasuhiro Uozumi (2008) Adv. Synth. Catal. 350: 
$1771-1775$

[7] J.M.Munoz-Molina, W.M.C. Sameera, E.Alvarez (2011) Inorg.Chem. 50: 2458-2467

[8] L.G. Hun, L. Nondek (1987) Coll. Czech. Chem. Commun., 52: 1758

[9] R. Davis, N.M.S. Khazal, T.E. Bitterwolf (1990) J. of Organomet. Chem., 397: 51

[10] W.J. Bland, R. Davis, J.L.A. Durrant (1985) J. of Organomet. Chem., 280: 397

[11] R.A.Gossage, L.A.Van de Kuil, G.Koten (1998) Acc.Chem.Res. 31: 423-431.

[12] L. Forti, F.Ghelfi, U.M.Pagnoni(1997) Tetrahedron. 53: $4419-4426$

[13] H. Uegaki, Y. Kotani, M. Kamigaito, M. Sawamoto (1997) Macromol., 30: 2249

[14] C. Granel, Ph. Dubois, R. Jérôme, Ph. Theyssié (1996) Macromol., 29: 8576.

[15] J.S. Wang, K. Matyjaszewski (1995) J. of Amer. Chem. Soc., 117: 5614

[16] M. Bialek, H. Cramail, A. Deffieux, S.M. Guilaume (2005) Europ. Polym. J., 41: 2678

[17] T.Pintauer (2010) Eur. J. Inorg. Chem. 2449-2460.

[18] T.Sasaki, C.Zhong, M.Tada, Y.Iwasawa (2005) Chem. Comm.:2506

[19] Berendsen G.E., Pikaart K.A., de Galan L. (1980) J. Liq. Chromatogr., 1437-1442

[20] V.V.Smirnov , I.G.Tarkhanova, A.I.Kokorin, V.I.Pergushov,
D.S.Tsvetkov (2005) Kinetics and Catalysis. 46: 65-71.

[21] V.V.Smirnov , I.G.Tarkhanova, A.I.Kokorin, M.G,Gantman, D.S.Tsvetkov (2005) Kinetics and Catalysis, 46: 72-76.

[22] V.V.Smirnov , I.G.Tarkhanova, A.I.Kokorin, D.S.Tsvetkov. (2005) Kinetics and Catalysis. 46: 861-866

[23] V.V.Smirnov, I.G.Tarkhanova, D.S.Tsvetkov (2007) Kinetics and Catalysis. 48: 271-275.

[24] J.A.Bertrand, E.Fujita, D.G.VanDerveer (1980) Inorg. Chem., 19: $2022-2028$

[25] J.Jezierska, T.Gowiak, A.Ozarowski, Y.Y.Yablokov. (1998) Inorg.Chim.Acta. 275-276: 28-36.

[26] Y.Nishida, S.Kida (1976) J. Inorg. Nucl. Chem., 38: 451-457

[27] J.E. Wertz, J.R. Bolton (1972) Electron Spin Resonance. Elementary theory and practical applications. McGraw Hill Book, Company, N.-Y.

[28] Goodman B. A., Raynor J. B. (1970) Adv. Inorg. Chem. Radiochem., 13: 135-162.

[29] Yu. N. Molin, K. M. Salikhov, K. I. Zamaraev, (1980) Spin Exchange. Springer-Verlag, Berlin

[30] N.M.Kabanov, A.I.Kokorin, V.B.Rogacheva and A.B.Zezin (1979) Polymer Sci. USSR, 21: 230-240

[31] L.S. Molochnikov, B.K Radionov (1995) Russ. J. Phys. Chem., 69: 836.

[32] Goldanskii V.I., Herber R.H. (1968) Chemical application of Mossbauer spectroscopy. Academic Press. N.-Y - London.

[33] I.S.Akhrem, A.V.Orlinkov, M.Vol'pin.(1993) J. Chem. Soc., Chem. Commun.:671-672. 